\title{
A mosaic of the Coma cluster of galaxies with XMM-Newton ${ }^{\star}$
}

\author{
U. G. Briel ${ }^{1}$, J. P. Henry ${ }^{1,2}$, D. H. Lumb ${ }^{3}$, M. Arnaud ${ }^{4}$, D. Neumann ${ }^{4}$, N. Aghanim ${ }^{5}$, R. Gastaud ${ }^{4}$, \\ J. P. D. Mittaz ${ }^{6}$, T. P. Sasseen ${ }^{7}$, and W. T. Vestrand ${ }^{8}$ \\ 1 Max-Planck-Institut für extraterrestrische Physik, 85748 Garching, Germany \\ 2 Institute for Astronomy, 2680 Woodlawn Drive, Honolulu, Hawaii 96822, USA \\ 3 XMM Science Operations Centre, Space Science Division ESTEC, Postbus299, 2200AG Noordwijk, \\ The Netherlands \\ ${ }^{4}$ CEA/DSM/DAPNIA Saclay, Service d'Astroph., L'Orme de Merisiers, Bât. 709, 91191 Gif-sur-Yvette, France \\ 5 IAS-CNRS, Bât. 121, Université Paris-Sud, 91405 Orsay Cedex, France \\ 6 Mullard Space Science Laboratory, Holmbury St. Mary, Surrey, RH6 6NT, UK \\ 7 Dept. of Physics, University of California at Santa Barbara, Santa Barbara, CA 93106, USA \\ 8 NIS-2, MS D436, Los Alamos National Laboratory, Los Alamos, NM 87545, USA
}

Received 6 October 2000 / Accepted 4 November 2000

\begin{abstract}
The Coma cluster of galaxies was observed with XMM-Newton in 12 partially overlapping pointings. We present here the resulting X-ray map in different energy bands and discuss the large scale structure of this cluster. Many point sources were found throughout the observed area, at least 11 of them are coincident with bright galaxies. We also give a hardness ratio map at the so far highest angular resolution obtained for a cluster of galaxies. In this map we found soft regions at the position of bright galaxies, little variation in the central 15 arcmin, but some harder regions north of the line NGC 4874 - NGC 4889.
\end{abstract}

Key words. coma - cluster of galaxies - intergalactic medium - large-scale structure of the Universe - X-rays

\section{Introduction}

The Coma cluster of galaxies was one of the performance verification targets of $X M M$-Newton. The aim was to verify XMM-Newton's ability to map large extended X-ray sources. This is not an easy task because errors can occur in several areas.

The telescope vignetting plays a major role when overlapping the same region of an extended source, measured at different off-axis angles. Because of the vignetting, the surface brightness of a region measured off axis seems to be lower than the same region measured on axis. The reason for that is the increasing loss of mirror collecting area with increasing off-axis angle, which in addition is energy dependent. By de-vignetting the different observations and normalizing the observations to the observing times the surface brightnesses of the same source regions are corrected and must show the same number of counts/ $\operatorname{arcmin}^{2} / \mathrm{s}$. If done correctly, detectoredges or regions of detector gaps should not be visible as

Send offprint requests to: U. G. Briel, e-mail: ugb@mpe.mpg.de

* Based on observations with XMM-Newton, an ESA Science Mission with instruments and contributions directly funded by ESA Member States and the USA (NASA). inhomogenities in the surface brightness of the merged observations.

The backgrounds in the image are problematic. The part of the background that is produced by particles inside the detector is not distinguishable from X-rays, is not vignetted, and is in general distributed homogeneously over the detector face (except for the lower end of the spectral range and around the detector intrinsic emission lines around $8 \mathrm{keV}$, see Briel et al. 2000). Hence this component must be subtracted before de-vignetting is applied. The extragalactic X-ray background is vignetted in the same manner as diffuse emission from an extended source and may therefore be de-vignetted in the same manner. But there is a third component in the background, the low energy protons, that are again indistinguishable from $\mathrm{X}$-rays. They come through the mirror system and show a different vignetting compared to X-rays (see Strüder et al. 2001). In addition, their spectrum and their flux is time variable. Because of the different vignetting, it is essential to subtract a "proton image" from the measured image before de-vignetting is applied, especially when the surface brightness of the proton induced background is in the same order of magnitude as the surface brightness of the extended source. 
Table 1. Journal of observations

\begin{tabular}{lllcccc}
\hline & Name of & & & \multicolumn{3}{c}{ pn-Camera observing times (ksec) } \\
\multicolumn{1}{c}{ Date } & Observation & RA(2000) & DEC(2000) & planned & performed & effective \\
\hline 2000 May 29 & Coma center & 125946.7 & 275700 & 15.0 & 22.7 & 15.4 \\
2000 June 21/22 & Coma 1 & 125647.7 & 272407 & 25.0 & 29.2 & 24.0 \\
2000 June 11/12 & Coma 2 & 125742.5 & 274338 & 25.0 & 34.8 & 34.7 \\
2000 June 27/28 & Coma 3 & 125832.2 & 272412 & 25.0 & 27.0 & 14.3 \\
2000 June 23 & Coma 4 & 130004.6 & 273124 & 25.0 & 20.0 & $(11.4)$ \\
2000 May 29 & Coma 5 & 125927.5 & 274653 & 20.0 & 25.0 & 10.0 \\
2000 June 12 & Coma 6 & 125850.0 & 275852 & 20.0 & 14.8 & 3.7 \\
2000 June 24 & Coma 7 & 125727.7 & 280841 & 25.0 & 8.1 & $(3.4)$ \\
not observed yet & Coma 8 & 130125.6 & 274353 & 26.0 & - & - \\
2000 June 12 & Coma 9 & 130032.7 & 275659 & 20.0 & 26.3 & 21.3 \\
2000 June 22 & Coma 10 & 125938.4 & 280740 & 20.0 & 26.3 & 16.6 \\
2000 June 24 & Coma 11 & 125836.5 & 282356 & 25.0 & 32.1 & 15.9 \\
not observed yet & Coma 12 & 130150.2 & 280928 & 25.0 & - & - \\
not observed yet & Coma 13 & 130036.5 & 282515 & 25.0 & - & - \\
2000 June 22 & Coma bkgd & 130137.0 & 271952 & 30.0 & 22.7 & 20.1 \\
\hline
\end{tabular}

Coma was chosen because it is a well studied source at all wavelength ranges. With a diameter of the main cluster, which is several times larger than the FOV of the EPIC cameras of about 26 arcmin, it is necessary to mosaic the cluster with $X M M-N e w t o n$ if the large scale structure and dynamics of the cluster are to be investigated. Although Coma was long believed to be the archetype of a relaxed cluster of galaxies, the X-ray image taken during the Rosat All Sky Survey revealed the complex large scale structure, especially the subgroup around NGC 4839 probably falling into the main cluster (Briel et al. 1992). Subsequent pointed observations with Rosat showed that Coma has irregular structure on different scales and was most probably formed by hierarchical clustering of several subunits, which are still visible in their X-ray emission (White et al. 1993). Observations with ASCA, and also the pointed Rosat observation, showed evidence of temperature structure in Coma (Honda et al. 1996; Briel \& Henry 1998; Donnelly et al. 1999; Watanabe et al. 1999). The interpretation of the temperature structure favors the ongoing merging of the subgroup around NGC 4839 with the main cluster, a scenario which is still debated.

In this letter we will present the result of merging 12 partially overlapping observations of the Coma cluster with the EPIC pn-CCD-detector on board XMM-Newton. In Sect. 2 we will describe the observations and present the data reduction and the method of merging the individual observations. In Sect. 3 we will discuss some of the many point sources in the image, the large scale structure of the X-ray surface brightness distribution, and the hardness ratio map of the inner cluster region.

\section{Observations and data reduction}

The region of the Coma cluster of galaxies was observed by the XMM-Newton Satellite (Jansen et al. 2001) during the performance verification phase. In Table 1 we show the journal of the observations with information on the pointing directions and observing times.

The two MOS-CCD cameras (Turner et al. 2001) were operated in their full-frame mode, which we believe to be well-calibrated for spectral analysis. However due to operational difficulties, less data coverage of the Coma region was obtained in these cameras. The pn-CCD camera (Strüder et al. 2001) was operated in the extended full frame mode (see the above papers for a description of the different modes). This latter mode provides potentially the best imaging performance of the pn-CCD camera, but at this date its spectral calibration is less reliable than that of the MOS observation. The analysis of this paper therefore contrasts with that of Arnaud et al. (2001), in that we concentrate more on the gross imaging properties and less on the spectral imaging. The hardness ratio map that we present, based on flux estimate in wide energy bands, is not sensitive to the remaining $\mathrm{PN}$ calibration uncertainties. For all observations, the medium filter was used (see Turner et al. 2001).

During the observations the proton induced background often varied, and in flares reached count rates up to several hundred counts/s in the energy band from 0.15 to $10 \mathrm{keV}$. To increase the signal-to-noise ratio and also to minimize the above described problems of the de-vignetting of residual proton induced events, we binned the $10-15 \mathrm{keV}$ events in $100 \mathrm{~s}$ time bins. From this light curve, times of bins with count rates above 100 counts/100 s were identified and excluded from further processing. We have chosen the high energy region because we do not expect many events from Coma in that band. This rate criterion gave in all but Coma 4, 6, and 7 reasonably long effective exposure times, which are given in Table 1. The rate criterion for Coma 4 and 7 were 200 counts and 800 counts respectively. We did include these high background times in order to get the mosaic of Coma as complete as possible. We do have to be careful with the 


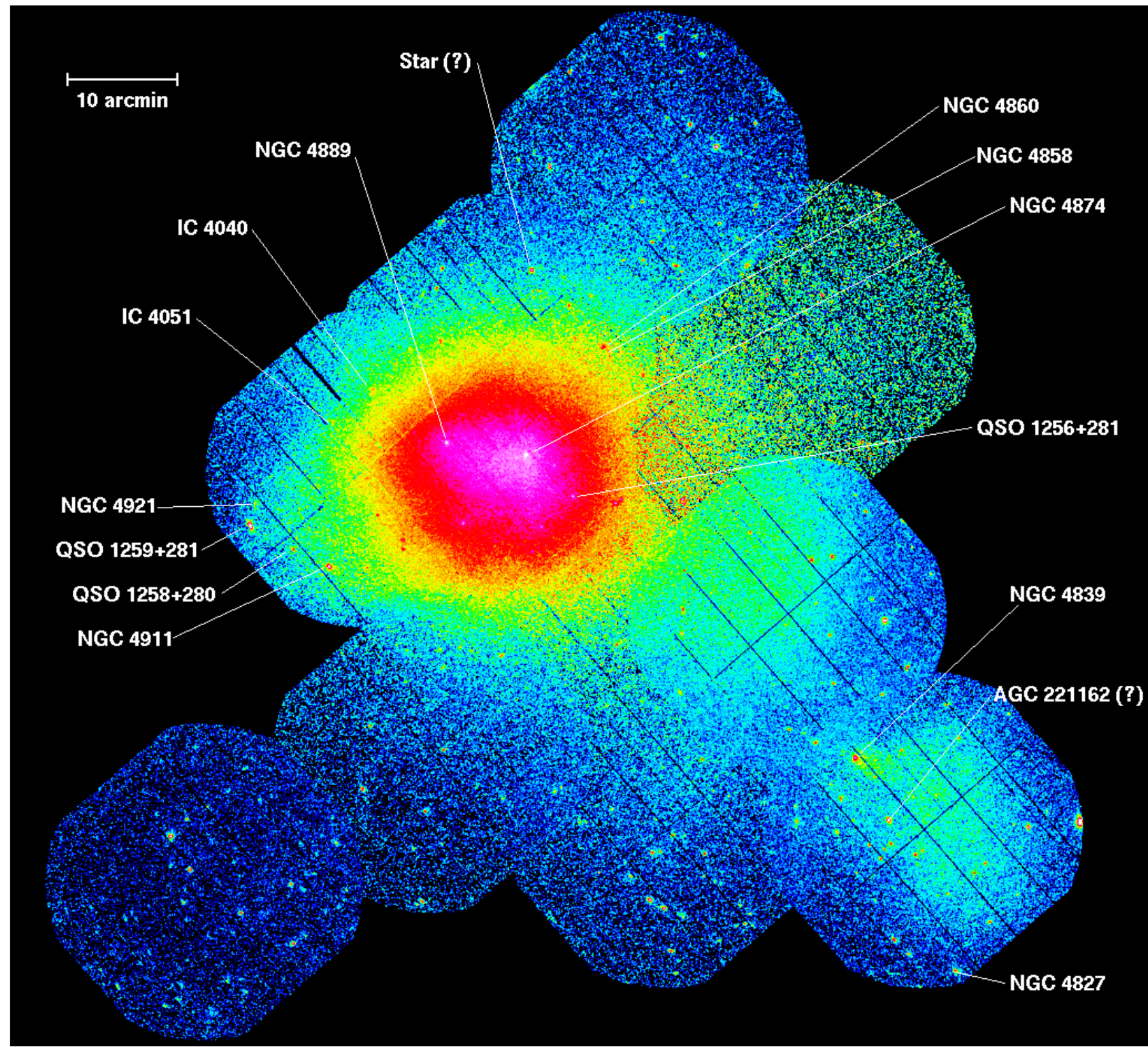

Fig. 1. The merged EPIC-pn image of the Coma cluster of galaxies in the 0.3 to $2.0 \mathrm{keV}$ energy band. Indicated are the tentative identifications of point sources

interpretation of those two fields. Since the Coma 6 field covers part of the center of the cluster, we did apply the stringent rate criterion, therefore $3.7 \mathrm{ksec}$ exposure time is usable, which is only $25 \%$ of the total time pointed at this position.

In addition to the above screening, we also excluded "warm" pixels from the images by checking each individual observation in detector coordinates and searching for single pixels with statistically significant higher numbers of events compared to adjacent pixels.

Images were produced in three different energy bands: $0.3-2 \mathrm{keV}, 2-5 \mathrm{keV}$ and $5-7.15 \mathrm{keV}$. We stopped at $7.15 \mathrm{keV}$ in order to avoid the strong detector intrinsic emission lines of $\mathrm{Ni}-\mathrm{K}, \mathrm{Cu}-\mathrm{K}$ and $\mathrm{Zn}-\mathrm{K}$, spread over approximately $2 \mathrm{keV}$. At a later time, when we learn more about the background, the higher energies will be included. An other handicap was the fact that, at the time of analysing the pn-data the correction files for the charge transfer inefficiency (CTI) were only available for the full frame mode (not the extended full frame mode used for our observations), so they were used to correct for the CTI. This effects the corrected amplitude of the events at the level of 5-25 eV depending on the energy and the position in the detector. But for producing images in the above bands, this systematic error is negligible. All valid events were used in the images. Valid events are all single events plus recombined split events, whose position is defined as the position of the pixel containing the maximum amplitude (see Dennerl et. al. 1999 for a detailed explanation). To minimize the gaps between CCDs, events at the border pixels are also included, although their amplitude could be slightly under estimated because of a possibly not recorded split companion. Again, for making images in wide energy bands, this systematic error is negligible. 


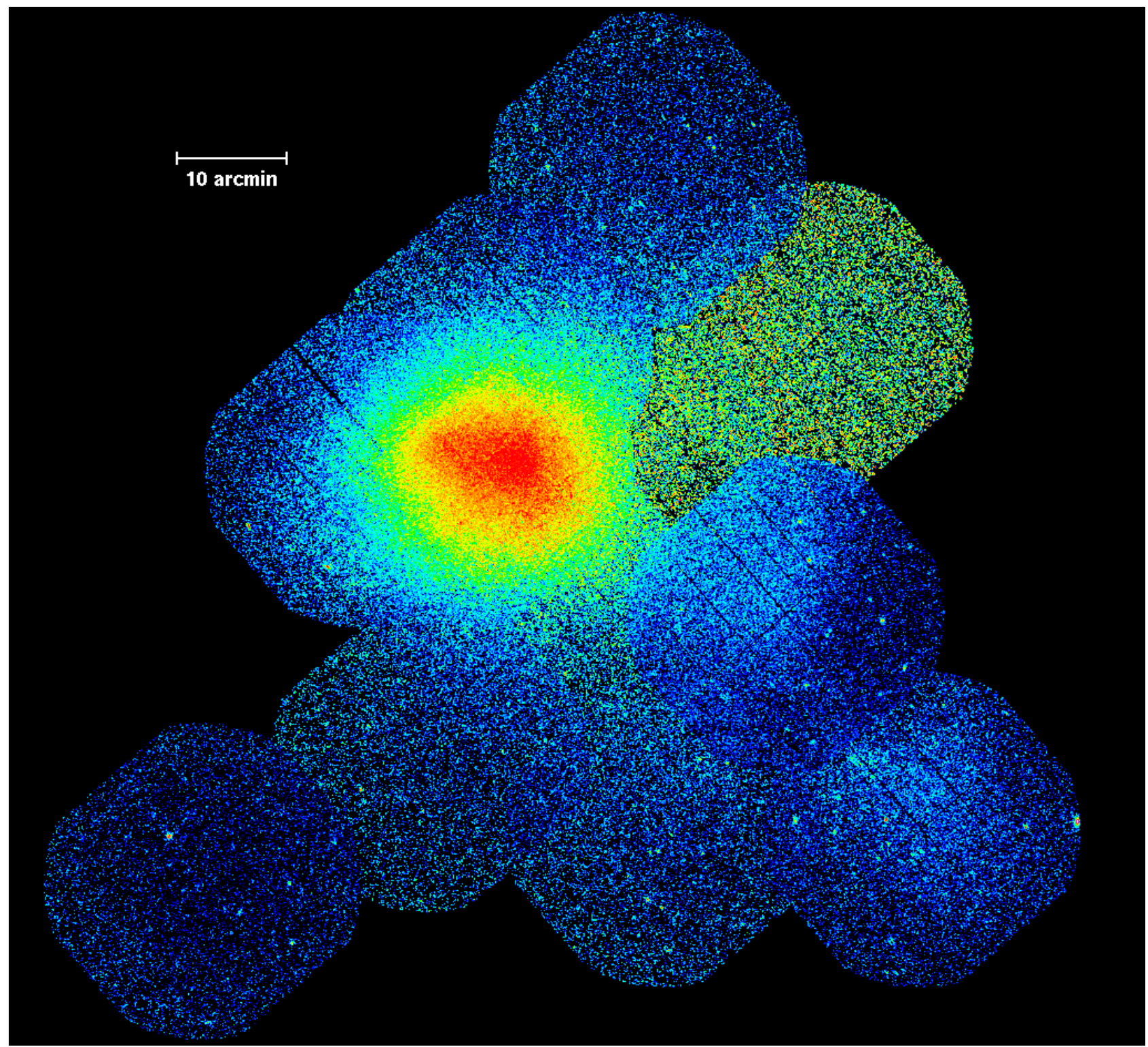

Fig. 2. The merged EPIC-pn image of the Coma cluster of galaxies in the 2.0 to $5.0 \mathrm{keV}$ energy band

In the same energy bands, and with the same selection criteria, background images were produces from a dedicated "background event file". This file contains $132.8 \mathrm{ksec}$ of pn-data accumulated from 5 other observations, like the Lockman Hole (Hasinger et al. 2001), containing no extended X-ray sources and only weak point sources. The single observations are between 13 and $33 \mathrm{ksec}$ long, spread over the 3 months CAL/PV phase of XMM-Newton. These data were also screened for flares and warm pixels, and obvious point sources were taken out. Comparing the single observations of the screened event files, the background rate in the energy band from 0.15 to $10 \mathrm{keV}$ is very stable, the systematic difference between the minimum and maximum rate is $8 \%$ of the mean. From each Coma image the corresponding background image, normalized using the effective observing times, was subtracted. Even though we restrict ourselves to observing times with "low" background, at a distance of 30 arcmin from the center of Coma the background is compareable to the surface brightness of Coma. At the center of Coma the background is $\sim 1.5 \%$ and $2.5 \%$ of Coma's surface brightness in the bands 0.3 to $2 \mathrm{keV}$ and 2 to $7.15 \mathrm{keV}$ respectively.

In a further step, exposure maps were produced for the different energy bands, also normalized to the corresponding effective observing times. These exposure maps contain the energy dependent vignetting.

The individual images in the lowest energy band were searched for point sources, and the positions of point sources in overlapping regions were then used to align and merge both the background-free X-ray images and the exposure maps. In a last step, the merged background free $\mathrm{X}$-ray images were divided by the corresponding merged exposure maps. The result is the surface brightness distribution of the Coma field in the different energy bands. In the Figs. 1 and 2 we show the $0.3-2.0 \mathrm{keV}$ 


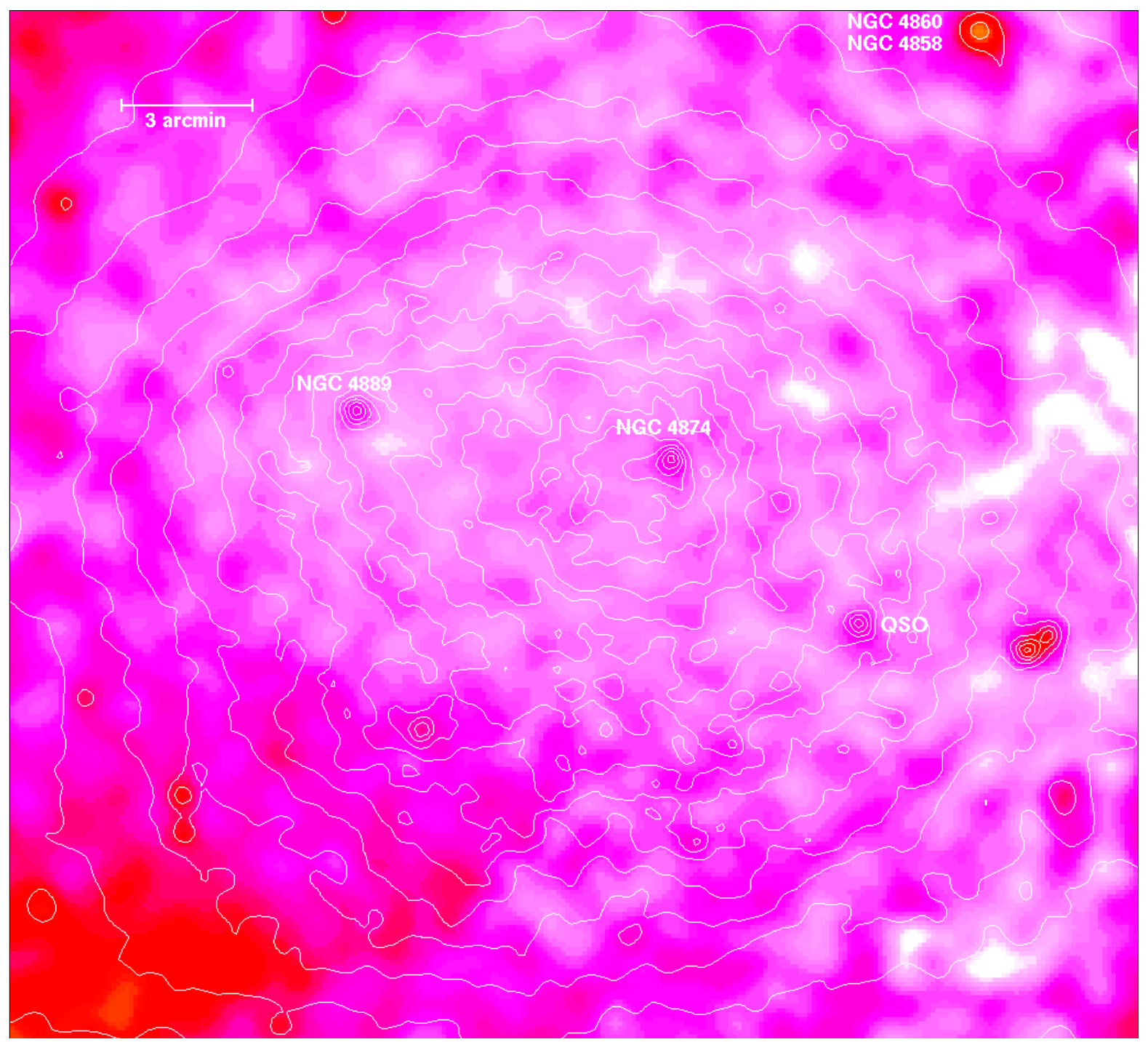

Hardness Ratio - Temperature Conversion

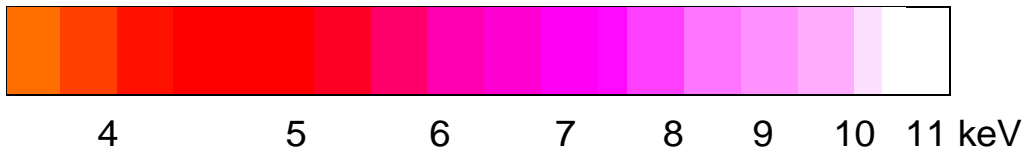

Fig. 3. The hardness ratio map of the inner Coma region in the energy bands from $0.3-2 \mathrm{keV}$ and $2-7.15 \mathrm{keV}$. The overlain contours are from the smoothed surface brightness distribution in the energy band from $0.3-2 \mathrm{keV}$

image and the $2-5 \mathrm{keV}$ image of Coma taken with the pn camera.

To visualize better the low surface brightness of the cluster we smoothed the images with two-dimensional Gaussian filters of variable sizes (with a sigma from $9 \operatorname{arcsec}$ to $96 \operatorname{arcsec}$ ). We used these images to look for spectral variations in the central Coma region. A hardness ratio map was produced by calculating the image $\mathrm{HR}=($ image $(2-7.15 \mathrm{keV})-$ image $(0.3-2 \mathrm{keV})) /($ image $(2-$ $7.15 \mathrm{keV})+$ image $(0.3-2 \mathrm{keV}))$. In Fig. 3 we show the inner $25.5 \times 23.3 \operatorname{arcmin}^{2}$ of this hardness ratio map. The overlain contours are from the smoothed surface brightness distribution in the $0.3-2 \mathrm{keV}$ band. We performed simulations with XSPEC, using a Raymond \& Smith model (with the fixed parameters $z=0.0232$, abundance $=0.25$ and $N_{\mathrm{H}}=8.9510^{19} \mathrm{~cm}^{-2}$ from Dickey \& Lockmann 1990) and taking into account our current understanding of the errors in the preliminary calibration of the extended full frame mode. With these simulations we converted the hardness ratios into "temperatures". The resulting conversion is indicated as color bar with the corresponding temperatures in Fig. 3.

The dynamic range of the hardness ratio in the map is of the order of $20 \%$, corresponding to a "temperature" variation of $\sim 5 \mathrm{keV}$. A systematic error of the background model of $10 \%$ would be neglectible in the inner 15 arcmin diameter. At larger radii the ratio would be 
different by $\sim 3 \%$, corresponding to a "temperature" difference of about $1 \mathrm{keV}$.

\section{Discussion}

The Coma cluster is the nearest very rich cluster of galaxies. It is probably the best studied cluster at all wavelengths and is one of the brightest extragalactic X-ray sources in the sky. Until now, the deepest X-ray observation of Coma is that of White et al. (1993) consisting of 4 exposures of about $19 \mathrm{ksec}$ each by the ROSAT PSPC. The EPIC pn observations reported here are of comparable length, thus are $\sim 8$ times deeper, while simultaneously extending over $\sim 3$ times wider energy range and with $\sim 4$ times better angular resolution.

Perhaps not surprisingly given the better spatial resolution and deeper observation, the most noticable difference between our XMM images in Figs. 1 and 2 compared to Fig. 2 of White et al. (1993) is the myriad of newly detected point-like sources. Dow \& White (1995) discuss the $\sim 25$ point sources in the ROSAT observations of Coma. There are at least 75 point sources in the smaller solid angle we have analyzed. We have made a first attempt to identify these sources by overlaying our X-ray map on an optical image of the region and then consulting NED when there appears to be a coincidence. Many bright galaxies seem to be X-ray sources including: NGC 4827, 4839, 4858, 4860, 4874, 4889, 4911, and 4921, and IC 4040 and 4051. However some faint galaxies, with magnitudes from 18 to 20 , could also be identifications based on positional coincidence. At least three quasars, QSO 1256+281, QSO $1258+280$, and QSO $1259+281$, are detected. However, the bulk of the sources are unidentified in our initial analysis.

The NGC 4911 and NGC 4839 groups contain concentrations of point sources at our sensitivity. Dow \& White (1995) and Neumann et al. (2001) discuss the particularly interesting morphology of the NGC 4839 group.

The large-scale diffuse emission in the soft band shown in Fig. 1 is very similar to the ROSAT observations of White et al. (1993) in the same band. The X-ray emission of the Coma cluster is lumpy near its center but becomes smoother at larger radii. An additional lump, apparent in the ROSAT observation but not discussed by White et al. (1993), is directly NE of NGC 4839. It may be yet another group on its way into the main cluster to the ENE. This lump may be preceeding the NGC 4839 group from the direction of A1367 since calculations of cluster growth indicate that the groups that merge to form clusters flow along filaments connecting the clusters. The large-scale diffuse hard emission is very similar to the soft, as Fig. 2 shows.

Because the calibration of the spectral response of the extended full frame mode of the pn-CCD is not yet available, we do not yet want to perform spatially resolved spectral fits. We can, however, make a hardness ratio map using wide bands, which we show in Fig. 3. While these maps sacrifice spectral details, such as temperatures and abundances, they are the highest angular $(\sim 40 \operatorname{arcsec})$ and spatial $\left(\sim 26 \mathrm{kpc}\right.$ for $\left.H_{0}=50 \mathrm{~km} \mathrm{~s}^{-1} \mathrm{Mpc}^{-1}\right)$ resolution spectral maps ever obtained for a cluster of galaxies.

Using the conversion from hardness ratio to temperature, given in Fig. 3, we have checked our resulting mean temperature in some regions which are known from other measurements. For the over-all temperature within a radius of 10 arcmin, centered on NGC 4874, we determined a temperature of $8.8 \mathrm{keV}$. The GINGA value is $8.21 \pm 0.09 \mathrm{keV}$ (Hughes et al. 1993), the ASCA value is 9. $\pm 0.6 \mathrm{keV}$ (Donnely et al. 1999) and the XMM-MOS value is $8.25 \pm 0.10 \mathrm{keV}$ (Arnaud et al. 2001). We agree to better than $10 \%$ with these previously determined values, which would be expected given our understanding of the uncertainties of the calibration and of the background model. In a further check, we determined the temperature around NGC 4874 within a radius of 0.5 arcmin and within a ring from 1 to 5 arcmin. Our result is 7 and $9.2 \mathrm{keV}$, the MOS result (Arnaud et al. 2001, Fig. 8) is 6.6 and $8.6 \mathrm{keV}$ respectively, again in agreement within $10 \%$. We found the same kind of agreement on other places, taking into account the large area of $3.5 \times 3.5 \mathrm{arcmin}^{2}$, used for the MOS data by Arnaud et al. (2001).

The large-scale description of the data in Fig. 3 agrees with Arnaud et al. (2001): there is cool gas to the southeast and hotter gas to the south-west and west. It agrees to some extent with Donnely et al. (1999), Watanabe et al. (1999) and Briel \& Henry (1998) investigations. In particular the south-east cool region was already identified by Donnely et al. (1999) and a hot region in the south-west/west direction, but somewhat further away, was found by Watanabe et al. (1999).

There are spectral variations on the smallest scales that we can measure. Softer emission is associated with many compact sources some of which are associated with galaxies as discussed previously. Although the smoothing of the original surface brightness distribution smears out the point sources to some extend, a significant drop in the radial temperature profile within 1 arcmin of NGC 4874 was also seen by Arnaud et al. (2001), Fig. 8. The Rosat temperature map of Briel \& Henry (1998) also shows cooler regions around the two central galaxies (although other cool regions in the center of their map are not confirmed). If these soft regions, associated with galaxies, are extended, we can postulate three possible explanations. First, they are local potential wells centered on cluster galaxies as is was discussed by Vikhlinin et al. (1994). The shallower potential wells could only trap lower temperature gas. Second, they are the interstellar emission that the cluster galaxy has been able to retain. Third, integrated emission from low mass X-ray binaries which Irwin \& Sarazin (1998) have discussed as the source of soft emission from elliptical galaxies.

Perhaps the most interesting small scale spectral structures are the isolated hard spots that do not appear to be associated with any obvious point-like X-ray emission. For the most part they are not associated with an optical object in NED. The five hard spots north of the 
NGC 4889 - NGC 4874 line may be the cause of the hot region in the same general location detected by Donnelly et al. (1999) in their ASCA observations. It seems that these hot spots are washed out in the temperature map of Arnaud et al. (2001) because of the larger $\left(3.5 \times 3.5 \mathrm{armin}^{2}\right)$ integration boxes. They might have been seen however in the even larger integration region of ASCA, if they are associated with variable sources like QSO's. But we can not entirely eliminate an instrumental cause for some of them because some are aligned parallel to the CCD orientation and on the boarder of the chips.

Acknowledgements. The XMM-Newton project is supported by the Bundesministerium für Bildung und Forschung/Deutsches Zentrum für Luft- und Raumfahrt (BMFT/DLR), the Max-Planck Society and the HeidenhainStiftung, and also by PPARC, CEA, CNES, and ASI. J. P. Henry thanks Prof. J. Trümper for the hospitality at the MPE. We thank the anonymous referee for useful comments, which improved the paper.

\section{References}

Arnaud, M., Aghanim, N., Gastaud, R., et al. 2001, A\&A, 365, L67

Briel, U. G., Henry, J. P., \& Böhringer, H. 1992, A\&A, 259, L31

Briel, U. G. \& Henry, J. P. 1998, in A Vision of an old Cluster:
Untangling Coma Berenices, ed. A. Mazure, F. Casoli, F. Durret, \& D. Gerbal (World Scientific Publishing), 170

Briel, U. G., Aschenbach, B., Balasini, M., et al. 2000, SPIE, 4012, 154

Dennerl, K., Briel, U. G., Haberl, F., et al. 1999, SPIE, 3765, 232

Dickey, J. M., \& Lockman, F. J. 1990, ARA\&A, 28, 215

Donnelly, R., Markevitch, M., Forman, W., et al. 1999, ApJ, 513,690

Dow, K. L., \& White, S. D. M. 1995, ApJ, 439, 113

Hasinger, G., Altieri, B., Arnaud, M., et al. 2001, A\&A, 365, L45

Honda, H., Hirayama, M., Watanabe, M., et al. 1996, ApJ, 473, L71

Hughes, J. P., Butcher, J. A., Stewart, G. C., et al. 1993, ApJ, 404,611

Irwin, J. A., \& Sarazin, C. L. 1998, ApJ, 499, 650

Jansen, F., Lumb, D., Altieri, B., et al. 2001, A\&A, 365, L1

Neumann, D. M., Arnaud, M., Gastaud, R., et al. 2001, A\&A, $365, \mathrm{~L} 74$

Strüder, L., Briel, U. G., Dennerl, K., et al. 2001, A\&A, 365, L18

Turner, M. J. L., Abbey, A., Arnaud, M., et al. 2001, A\&A, 365, L27

Vikhlinin, A., Forman, W., \& Jones, C. 1994, ApJ, 435, 162

Watanabe, M., Yamashita, K., Furuzawa, A., et al. 1999, ApJ, 527,80

White, S., Briel, U. G., \& Henry, J. P. 1993, MNRAS, 261, L8 